\title{
AN INVESTIGATION OF STRESSES AND DEFORMATION STATES OF CLAMPED ROTATING FUNCTIONALLY GRADED DISKS
}

\author{
Amit Kumar Thawait, Lakshman Sondhi \\ Department of Mechanical Engineering, Shri Shankaracharya Technical Campus (SSGI), Bhilai, India \\ e-mail: amkthawait@gmail.com; lsondhii@gmail.com
}

Shubhashis Sanyal, Shubhankar Bhowmick

Department of Mechanical Engineering, National Institute of Technology (NIT), Raipur, India

\begin{abstract}
The present study deals with the linear elastic analysis of variable thickness rotating disks made of functionally graded materials (FGMs) by the finite element method. The disks have radially varying material properties according to an exponential law, which is achieved by the element based grading of the material properties on the meshed domain. The results are reported for three types of thickness profiles, namely, uniform, linearly varying and concave thickness, having their mass constant. The disks are subjected to the clamped boundary condition at the inner surface and the free boundary condition at the outer surface. The obtained results show that in a variable thickness rotating disk, deformation and stresses are less as compared to the uniform thickness disk.
\end{abstract}

Keywords: functionally graded material (FGM), linear elastic analysis, annular rotating disk, variable thickness rotating disk, finite element method (FEM)

\section{Introduction}

Functionally graded materials (FGMs) are special composite materials that have continuous and smooth spatial variations of physical and mechanical properties. Functionally graded components, in recent years, are widely used in space vehicles, aircrafts, nuclear power plants and many other engineering applications. Rotating disks, made up of such a FGM are widely used in the field of marine, mechanical and aerospace industry including gas turbines, gears, turbo-machinery, etc. The stresses due to centrifugal load in rotating components have important effects on their strength and safety. Thus, control and optimization of stress and displacement fields can help one to reduce the overall payload in industries. Optimization of the stress to strength ratio is done by varying the material property and thickness of the disk. Disks made up of functionally graded materials and of variable thickness, have significant stress reduction over the disks made up of homogeneous materials and of uniform thickness. Therefore, a higher limit speed and higher pressure is permissible for FGM disks.

A few researchers have reported works on analysis of FGM disks, plates, shells, beams and bars by analytical and finite element methods. Eraslan (2003) obtained analytical solutions for the elastic plastic stress distribution in rotating variable thickness annular disks. Thickness of the disks had parabolic variation and the analysis was based on the Tresca's yield criterion. Bayat et al. (2009) reported work on analysis of a variable thickness FGM rotating disk. Material properties varied according to power law and the disk was subjected to both the mechanical and thermal loads. Afsar and Go (2010) analyzed a rotating FGM circular disk subjected to mechanical as well as thermal load by the finite element method. The disk had exponentially varying 
material properties in the radial direction. The inner surface was made up of $\mathrm{Al}_{2} \mathrm{O}_{3}$ having fix boundary condition and the outer surface was made up of $\mathrm{Al}$ having free boundary condition. The disk was subjected to a thermal load along with centrifugal load due to non uniform temperature distribution. The axisymmetric problem was formulated in terms of a second order ordinary differential equation and was solved by the finite element method. Callioglu et al. (2011a) analyzed functionally a graded rotating annular disk subjected to internal pressure and various temperature distributions such as uniform temperature, linearly increasing and decreasing temperatures in the radial direction. An analytical thermoelasticity solution for a disk made of functionally graded materials (FGMs) was presented by Callioglu (2011). Bayat et al. (2011) investigated displacement and stress behavior of a functionally graded rotating disk of variable thickness by a semi analytical method. Radially varying one dimensional FGM was taken and material properties varied according to a power law and the Mori-Tanaka scheme. A disk subjected to centrifugal load was analyzed for the fixed boundary condition at the inner surface and the free boundary condition at the outer surface. The results were reported for both metal-ceramic and ceramicetal disks and, a comparison was made for uniform and variable thickness disks. Callioglu et al. (2011b) analyzed thin FGM disks. Density and modulus of elasticity of them varied according to a power law in an FGM of aluminum ceramic. The effect of the grading parameter on displacement and stresses was investigated. Sharma et al. (2012) worked on the analysis of stresses, displacements and strains in a thin circular functionally graded material (FGM) disks by the finite element method. The disk were subjected to mechanical as well as thermal loads. Ali et al. (2012) reported a study on the elastic analysis of two sigmoid FGM rotating disks. Metal-ceramic-metal disks were analyzed for both uniform and variable thickness disks and effect of grading index on the displacement and stresses was investigated. Nejad et al. (2013) found a closed-form analytical solution for an exponentially varying FGM disk which was subjected to internal and external pressure.

In his recent work, Zafarmand and Hassani (2014) worked on elastic analysis of two-dimensional functionally graded rotating annular and solid disks with variable thickness. Axisymmetric conditions were assumed for the two-dimensional functionally graded disk and the graded finite element method (GFEM) was applied to solve the equations. Rosyid et al. (2014) worked on finite element analysis of nonhomogeneous rotating disk with arbitrarily variable thickness. Three types of grading laws, namely, power law, sigmoid and exponential distribution laws were considered for the volume fraction distributions. The work included parametric studies performed by varying volume fraction distributions and boundary conditions. Zafarmand and Kadkhodayan (2015) investigated a nonlinear elasticity solution of functionally graded nanocomposite rotating thick disks with variable thickness reinforced with single-walled carbon nanotubes (SWCNTs). The derived governing nonlinear equations were based on the axisymmetric theory of elasticity with the geometric nonlinearity in axisymmetric complete form and were solved by a nonlinear graded finite element method (NGFEM). The nonlinear graded finite element method (NGFEM) used in that study was based on the Rayleigh-Ritz energy formulation with the Picard iterative scheme. The results were reported for four different thickness profiles, namely, constant, linear, concave and convex.

In the present research work, stress and deformation analysis of annular rotating FGM disks is reported, which is based on the element based grading of material properties. Uniform as well as variable thickness disks, made of exponentially varying FGMs, are analyzed. The disks are subjected to centrifugal body load and have the clamped boundary condition at the inner surface and the free boundary condition at the outer surface. The finite element method based on the principle of stationary total potential is used to analyze disks. Numerical results are evaluated for a uniform, linear varying thickness profile and concave thickness profile disks, and the effect of the thickness parameter on the deformation and stresses is investigated. 


\section{Geometric modeling}

For an annular disk, the governing equation of radially varying thickness is assumed as

$$
h(r)=h_{0}\left[1-q\left(\frac{r-a}{b-a}\right)^{m}\right]
$$

where $a$ and $b$ are the inner and outer radii, $h(r)$ and $h_{0}$ are half of the thickness at the radius $r$ and at the root of the disk, respectively. Symbols $m$ and $q$ are geometric parameters that control the thickness profiles of the disk. For a uniform thickness disk $q$ is taken as zero and for a variable thickness disk, $q>0$ (Fig. 1b). The value of $h_{0}$ is calculated for each thickness profile to get constant mass for all thickness profile disks.

(a)

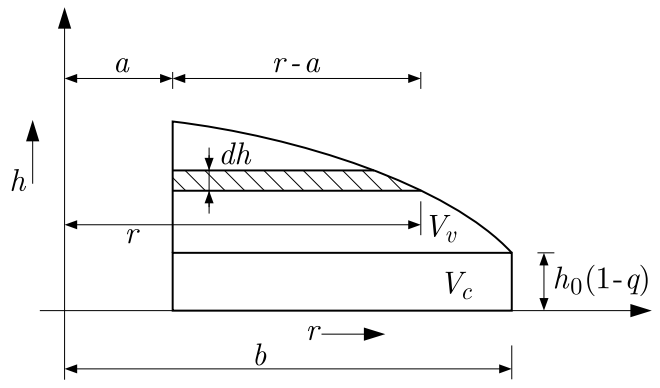

(b)

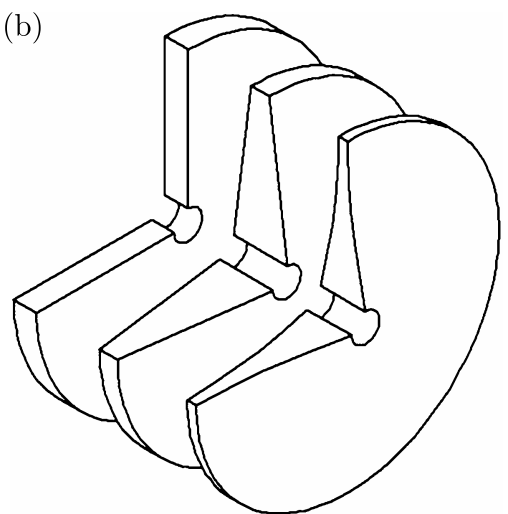

Fig. 1. (a): Geometrical parameters of the variable thickness disk, (b) disks of varying thickness; sectional isometric view

\subsection{Calculation of $h_{0}$ for the variable thickness profile}

Figure 1a shows half of the cross section of the variable thickness disk. The symbol $V_{c}$ denotes the volume of the disk till height $h_{0}(1-q)$ and the symbol $V_{v}$ is the volume from $h_{0}(1-q)$ to height at the inner radius. The symbol $V$ denotes the total volume of the disk

$$
V_{c}=\pi\left(b^{2}-a^{2}\right)(1-q) h_{0} \quad V_{v}=\int_{(1-q) h_{0}}^{h_{0}} \pi\left(r^{2}-a^{2}\right) d h \quad V=2\left(V_{c}+V_{v}\right)
$$

Since mass of the variable thickness disk equals mass of the uniform thickness disk

$$
\rho_{1} V=\rho_{u} V_{u}
$$

where $\rho_{1}$ and $\rho_{u}$ are densities of variable thickness and uniform thickness disks, respectively. The symbol $V_{u}$ is the volume of the uniform thickness disk. Assuming $h_{0}$ of the uniform thickness disk as $h_{u}, V_{u}$ is obtained as

$$
V_{u}=2 \pi\left(b^{2}-a^{2}\right) h_{u}
$$

Since density is independent of thickness, it is constant for all thickness profiles, therefore equation $(2.3)$ reduces to

$$
V=V_{u}
$$


putting the values of $V, V_{u}, V_{c}$, and $V_{v}$ into equation (2.5)

$$
\pi\left(b^{2}-a^{2}\right)(1-q) h_{0}+\int_{(1-q) h_{0}}^{h_{0}} \pi\left(r^{2}-a^{2}\right) d h=\pi\left(b^{2}-a^{2}\right) h_{u}
$$

Substituting the value of $r$ from equation (2.1) to equation (2.6) and solving the resulting equation for given thickness of the uniform disk and different values of $m$, we obtain value of $h_{0}$ for different thickness profiles.

\section{Material modeling}

Young's modulus and density of the disk are assumed to vary exponentially along the radial direction as (Afsar and Go, 2010):

$$
\begin{array}{lll}
E(r)=E_{0} \mathrm{e}^{\beta r} & \rho(r)=\rho_{0} \mathrm{e}^{\gamma r} & E_{0}=E_{A} \mathrm{e}^{-\beta a} \\
\rho_{0}=\rho_{A} \mathrm{e}^{-\gamma a} & \gamma=\frac{1}{a-b} \ln \frac{\rho_{A}}{\rho_{B}} & \beta=\frac{1}{a-b} \ln \frac{E_{A}}{E_{B}}
\end{array}
$$

where $E(r)$ and $\rho(r)$ are modulus of elasticity and density at the radius $r ; E_{A}, E_{B}$ and $\rho_{A}, \rho_{B}$ are modulus of elasticity and density at the inner and outer radius, respectively.

\section{Finite element modeling}

The rotating disk, being thin, is modeled as a plane stress axisymmetric problem. Using quadratic quadrilateral element, the displacement vector $\mathbf{u}$ can be obtained as (Seshu, 2003)

$$
\mathbf{u}=\mathbf{N} \delta
$$

where $\mathbf{u}$ is the element displacement vector, $\mathbf{N}$ is the matrix of quadratic shape functions and $\boldsymbol{\delta}$ is the nodal displacement vector

$$
\mathbf{N}=\left[\begin{array}{llll}
N_{1} & N_{2} & \ldots & N_{8}
\end{array}\right] \quad \boldsymbol{\delta}=\left\{\begin{array}{lllll}
u_{1} & u_{2} & \ldots & \ldots & u_{8}
\end{array}\right\}^{\mathrm{T}}
$$

In natural co-ordinates, the shape functions are given as

$$
\begin{aligned}
N_{1} & =\frac{1}{4}(1-\xi)(1-\eta)(-1-\xi-\eta) & N_{2} & =\frac{1}{4}(1+\xi)(1-\eta)(-1+\xi-\eta) \\
N_{3} & =\frac{1}{4}(1+\xi)(1+\eta)(-1+\xi+\eta) & N_{4} & =\frac{1}{4}(1-\xi)(1+\eta)(-1-\xi+\eta) \\
N_{5} & =\frac{1}{2}\left(1-\xi^{2}\right)(1-\eta) & N_{6} & =\frac{1}{2}(1+\xi)\left(1-\eta^{2}\right) \\
N_{7} & =\frac{1}{2}\left(1-\xi^{2}\right)(1+\eta) & N_{8} & =\frac{1}{2}(1-\xi)\left(1-\eta^{2}\right)
\end{aligned}
$$

The strain components are related to elemental displacement components as

$$
\begin{aligned}
& \varepsilon=\left\{\begin{array}{ll}
\varepsilon_{r} & \varepsilon_{\theta}
\end{array}\right\}^{\mathrm{T}}=\left\{\begin{array}{ll}
\frac{\partial u}{\partial r} & \frac{u}{r}
\end{array}\right\}^{\mathrm{T}} \\
& \left\{\begin{array}{ll}
\frac{\partial u}{\partial r} & \frac{u}{r}
\end{array}\right\}^{\mathrm{T}}=\mathbf{B}_{1}\left\{\begin{array}{lll}
\frac{\partial u}{\partial r} & \frac{\partial u}{\partial z} & \frac{u}{r}
\end{array}\right\}^{\mathrm{T}}
\end{aligned}
$$


where $\varepsilon_{r}$ and $\varepsilon_{\theta}$ are radial and tangential strains, respectively. By transforming the global co-ordinates into natural co-ordinates $(\xi \eta)$, we obtain

$$
\begin{aligned}
& \left\{\begin{array}{lll}
\frac{\partial u}{\partial r} & \frac{\partial u}{\partial z} & \frac{u}{r}
\end{array}\right\}^{\mathrm{T}}=\mathbf{B}_{2}\left\{\begin{array}{lll}
\frac{\partial u}{\partial \xi} & \frac{\partial u}{\partial \eta} & \frac{u}{r}
\end{array}\right\}^{\mathrm{T}} \\
& \left\{\begin{array}{llll}
\frac{\partial u}{\partial \xi} & \frac{\partial u}{\partial \eta} & \frac{u}{r}
\end{array}\right\}^{\mathrm{T}}=\mathbf{B}_{3}\left\{\begin{array}{llll}
u_{1} & u_{2} & \ldots & u_{8}
\end{array}\right\}^{\mathrm{T}}
\end{aligned}
$$

The above elemental strain-displacement relationships can be written as

$$
\varepsilon=\mathbf{B} \boldsymbol{\delta}^{e}
$$

where $\mathbf{B}$ is the strain-displacement relationship matrix which contains derivatives of the shape functions. For a quadratic quadrilateral element, it is calculated as

$$
\mathbf{B}=\mathbf{B}_{1} \mathbf{B}_{2} \mathbf{B}_{3}
$$

and

$$
\mathbf{B}_{1}=\left[\begin{array}{lll}
1 & 0 & 0 \\
0 & 0 & 1
\end{array}\right] \quad \mathbf{B}_{2}=\left[\begin{array}{ccc}
\frac{J_{22}}{|\mathbf{J}|} & \frac{-J_{12}}{|\mathbf{J}|} & 0 \\
\frac{-J_{21}}{|\mathbf{J}|} & \frac{J_{11}}{|\mathbf{J}|} & 0 \\
0 & 0 & 1
\end{array}\right]
$$

where $\mathbf{J}$ is the Jacobian matrix used to transform the global co-ordinates into natural co-ordinates. It is given as

$$
\mathbf{J}=\left[\begin{array}{cc}
\sum_{i=1}^{8} \frac{\partial N_{i}}{\partial \xi} r_{i} & \sum_{i=1}^{8} \frac{\partial N_{i}}{\partial \xi} z_{i} \\
\sum_{i=1}^{8} \frac{\partial N_{i}}{\partial \eta} r_{i} & \sum_{i=1}^{8} \frac{\partial N_{i}}{\partial \eta} z_{i}
\end{array}\right] \quad \mathbf{B}_{3}=\left[\begin{array}{cccc}
\frac{\partial N_{1}}{\partial \xi} & \frac{\partial N_{2}}{\partial \xi} & \ldots & \frac{\partial N_{8}}{\partial \xi} \\
\frac{\partial N_{1}}{\partial \eta} & \frac{\partial N_{2}}{\partial \eta} & \ldots & \frac{\partial N_{8}}{\partial \eta} \\
\frac{N_{1}}{r} & \frac{N_{2}}{r} & \ldots & \frac{N_{8}}{r}
\end{array}\right]
$$

From Hooke's law, the components of stresses in the radial and circumferential direction are related to the components of total strain as

$$
\varepsilon_{r}=\frac{1}{E}\left(\sigma_{r}-\nu \sigma_{\theta}\right) \quad \varepsilon_{\theta}=\frac{1}{E}\left(\sigma_{\theta}-\nu \sigma_{r}\right)
$$

By solving the above equations, the stress-strain relationship can be obtained as follows

$$
\sigma_{r}=\frac{E(r)}{(1-\nu)^{2}}\left(\varepsilon_{r}+\nu \varepsilon_{\theta}\right) \quad \sigma_{\theta}=\frac{E(r)}{(1-\nu)^{2}}\left(\varepsilon_{\theta}+\nu \varepsilon_{r}\right)
$$

In the standard finite element matrix notation, the above stress strain relations can be written as

$$
\boldsymbol{\sigma}=\mathbf{D}(r) \boldsymbol{\varepsilon}
$$

where

$$
\boldsymbol{\sigma}=\left\{\begin{array}{ll}
\sigma_{r} & \sigma_{\theta}
\end{array}\right\}^{\mathrm{T}} \quad \mathbf{D}(r)=\frac{E(r)}{(1-\nu)^{2}}\left[\begin{array}{ll}
1 & \nu \\
\nu & 1
\end{array}\right] \quad \boldsymbol{\varepsilon}=\left\{\begin{array}{ll}
\varepsilon_{r} & \varepsilon_{\theta}
\end{array}\right\}^{\mathrm{T}}
$$


Upon rotation, the disk experiences a body force which under constrained boundary results in deformation and stores internal strain energy $U$

$$
U=\frac{1}{2} \int_{V} \varepsilon^{\mathrm{T}} \boldsymbol{\sigma} d v
$$

The work potential due to body force resulting from centrifugal action is given by

$$
V=-\int_{V} \boldsymbol{\delta}^{\mathrm{T}} \mathbf{q}_{v} d v
$$

Upon substituting Eq. (4.4) and (4.9) into Eq. (4.10) and Eq. (4.11), the elemental strain energy and work potential are given by

$$
U^{e}=\int_{V} \pi r h_{r} \boldsymbol{\delta}^{e \mathrm{~T}} \mathbf{B}^{\mathrm{T}} \mathbf{D}(r) \mathbf{B} \boldsymbol{\delta}^{e} d r \quad V^{e}=-2 \int_{V} \pi r h_{r} \boldsymbol{\delta}^{e \mathrm{~T}} \mathbf{N}^{\mathrm{T}} \mathbf{q}_{v} d r
$$

For a disk rotating at $\omega[\mathrm{rad} / \mathrm{s}]$, the body force vector for each element is given by

$$
\mathbf{q}_{v}=\left\{\begin{array}{c}
\rho(r) \omega^{2} r \\
0
\end{array}\right\}
$$

The total potential of the element is obtained from Eqs. (4.12)

$$
\pi_{p}^{e}=\frac{1}{2} \boldsymbol{\delta}^{e \mathrm{~T}} \mathbf{K}^{e} \boldsymbol{\delta}^{e}-\boldsymbol{\delta}^{e \mathrm{~T}} \mathbf{f}^{e}
$$

Here, defining the element stiffness matrix $\mathbf{K}^{e}$ and the element load vector $\mathbf{f}^{e}$ as

$$
\mathbf{K}^{e}=2 \int_{V} \pi r h_{r} \mathbf{B}^{\mathrm{T}} \mathbf{D}(r) \mathbf{B} d r \quad \mathbf{f}^{e}=2 \int_{V} \pi r h_{r} \mathbf{N}^{\mathrm{T}} \mathbf{q}_{v} d r
$$

In FEM, the functional grading is popularly carried out by assigning the average material properties over a given geometry followed by adhering the geometries, thus resulting into layered functional grading of material properties. The downside of this approach is that it yields singular field variable values at the boundaries of the glued geometries. To get better results, it is an established practice to divide the total geometry into very fine geometries. However, a better approach is to assign the average material properties to the elements of mesh of the single geometry. This is, in other words, better described as assigning material properties to the finite elements instead of geometry. In Eq. (4.9), the matrix $\mathbf{D}(r)$, being a function of $r$, is calculated numerically at each node, and this yields continuous material property variation throughout the geometry. The element matrices are then assembled to give the global stiffness matrix and the global load vector, respectively.

The element based grading of the material property yields an appropriate approach of functional grading as the shape functions in the elemental formulations being co-ordinate functions make it easier to implement the same (Kim and Paulino, 2002)

$$
\phi^{e}=\sum_{i=1}^{8} \phi_{i} N_{i}
$$

where $\phi_{e}$ is the material property of the element, $\phi_{i}$ is the material property at the node $i$, and $N_{i}$ is the shape function. 
Total potential energy of the disk is given by

$$
\pi_{p}=\sum \pi_{p}^{e}=\frac{1}{2} \boldsymbol{\delta}^{\mathrm{T}} \mathbf{K} \boldsymbol{\delta}-\boldsymbol{\delta}^{\mathrm{T}} \mathbf{F}
$$

where $\mathbf{K}$ is global stiffness matrix, $\mathbf{F}$ isglobal load vector

$$
\mathbf{K}=\sum_{n=1}^{N} \mathbf{K}^{e} \quad \mathbf{F}=\sum_{n=1}^{N} \mathbf{f}^{e}
$$

and $N$ is number of elements.

Using the Principle of Stationary Total Potential (PSTP), the total potential is set to be stationary with respect to small variation in the nodal degree of freedom, that is

$$
\frac{\partial \pi_{p}}{\partial \boldsymbol{\delta}^{\mathrm{T}}}=\mathbf{0}
$$

From above, the system of simultaneous equations is obtained as follows

$$
\mathbf{K} \delta=\mathbf{F}
$$

\section{Results and discussion}

\subsection{Validation}

A numerical problem of reference (Bayat et al., 2011) is modeled and analyzed, and the comparison is shown in Fig. 2 for the validation purpose. In the reference rotating disks having uniform and concave thickness, the profiles are analyzed. Gradation of the material properties is done by the Mori-Tanaka scheme and comparison is made for $n=0$ for ceramic-metal and metal-ceramic disks.

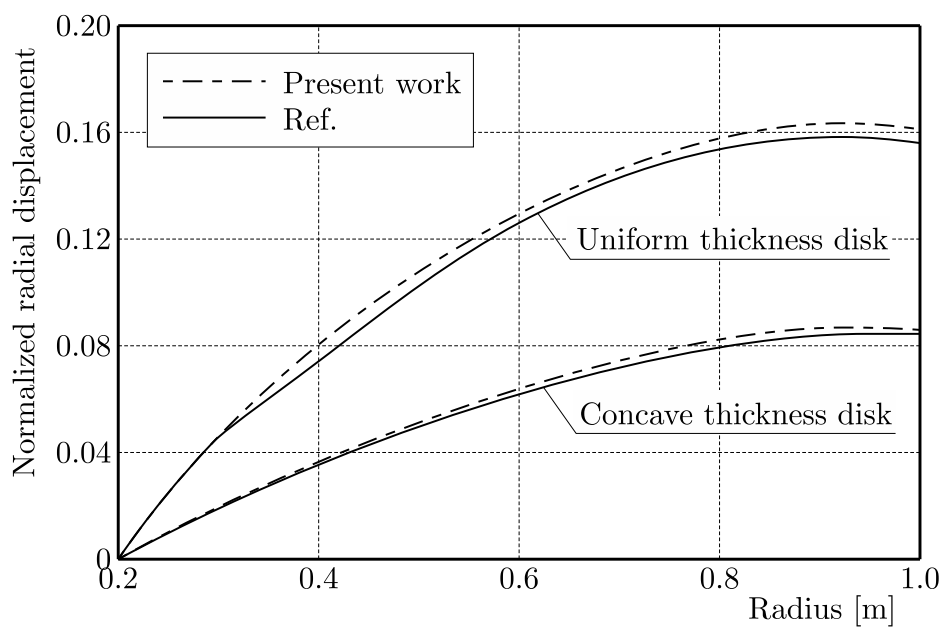

Fig. 2. Comparison of the results of the current work with the reference ones (Bayat et al., 2011)

\subsection{Numerical results}

Rotating annular disks made of aluminum and alumina ceramic are analyzed, and the distribution of resulting displacement and stresses are presented for different thickness profiles. The material properties are graded according to an exponential law as discussed in Section 3. Figures $3 \mathrm{a}$ and 3b show the distribution of Young's modulus and density of the exponential 

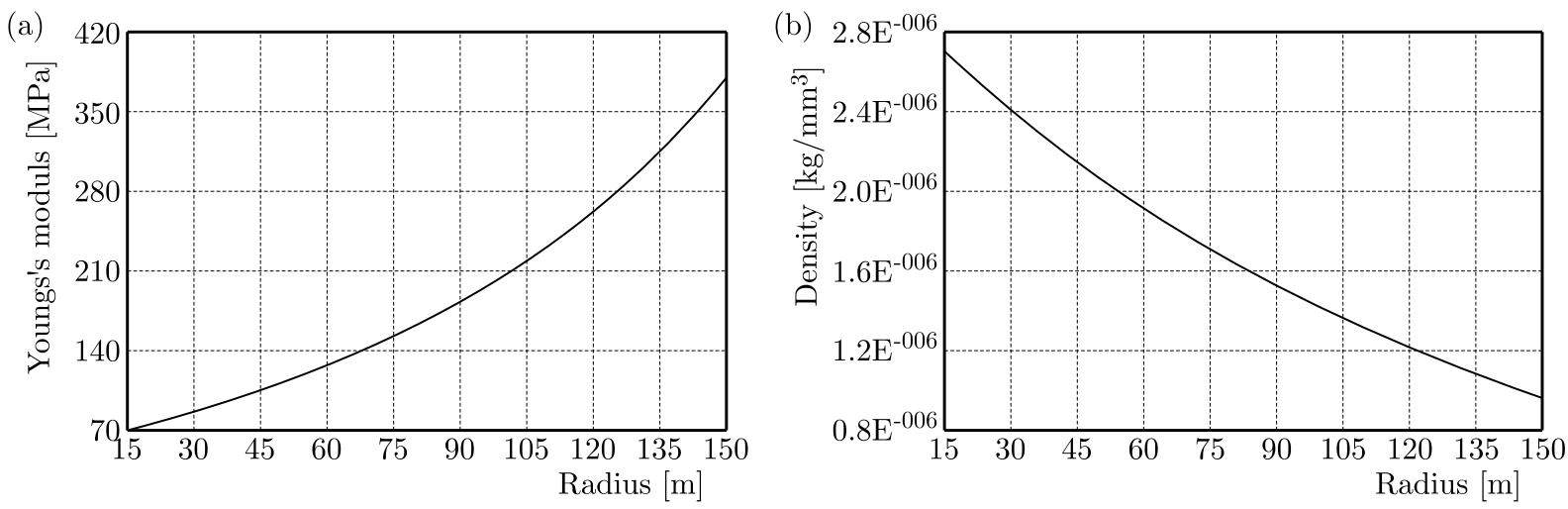

Fig. 3. Radial distribution of Young's modulus (a) and of density (b)

FGM considered here. The properties of aluminum and alumina ceramic are given as (Afsar and Go, 2010): $E_{A l}=71.0 \mathrm{MPa}, E_{c e r}=380 \mathrm{MPa}, \rho_{A l}=2.7 \mathrm{~g} / \mathrm{cm}^{3}, \rho_{c e r}=0.96 \mathrm{~g} / \mathrm{cm}^{3}$ and $\vartheta=0.3$.

In the present numerical problem, the inner diameter of the disks is taken as $15 \mathrm{~mm}$ and outer diameter $150 \mathrm{~mm} ; q=0.7$ and $h_{u}$ is taken as $5 \mathrm{~mm}, h_{0}$ for linear and concave thickness profiles are obtained as $9.0164 \mathrm{~mm}$ and $10.9416 \mathrm{~mm}$ from Eq. (2.6) for $m=1$ and 0.5 , respectively. The disks have an angular velocity of $100 \mathrm{rad} / \mathrm{s}$.

Figures 4 and 5 show the distribution of radial displacement, radial stress, tangential stress and von Mises stress, respectively, along the radial direction. It is observed that the uniform thickness disk has highest deformation and stresses as compared to the linear thickness profile and concave thickness profile disk. Stresses and deformations are less near the inner radius and higher near the outer radius for the concave thick disk as compared to the linear disk. This is because of the fact that the concave thick disk has greater thickness near the root as compared to the linear thick disk. The radial displacement is minimum, that is zero at the inner surface and the radial stress is zero at the outer surface for all thickness profiles, which confirms the clamped boundary condition at the inner surface and the free boundary condition at the outer surface applied on the disks. The tangential stress is maximum at the outer radius for all thickness profiles, which corresponds to the complete ceramic material. Since ceramics have low tensile strength, to withstand higher stresses at higher speeds, sufficient thickness at the outer radius should be provided, which means that the value of geometric parameter $q$ in equation (2.1) should be taken smaller at higher speeds. Further it can be seen that the radial stress is higher as compared to the tangential and von Mises stresses for all thickness profiles. Therefore, it is suggested that during designing of rotating disks, the radial stress should be taken as the critical limit stress, and the concave thickness profile should be selected.
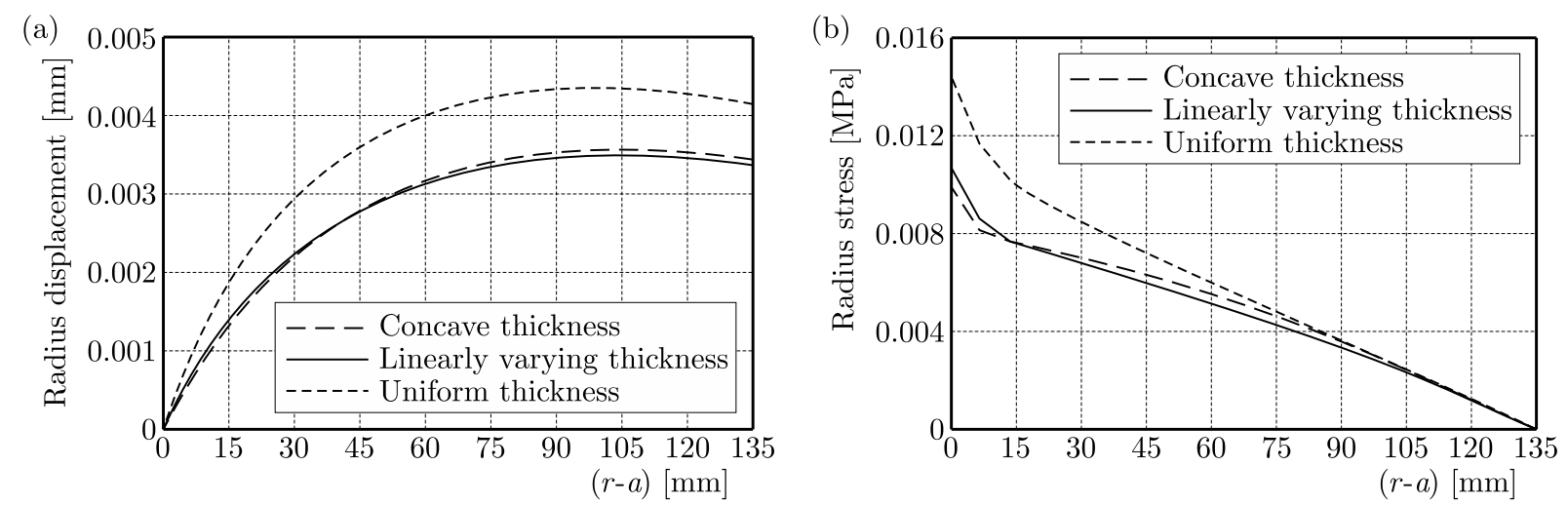

Fig. 4. Distribution of: (a) radial displacements, (b) radial stress 

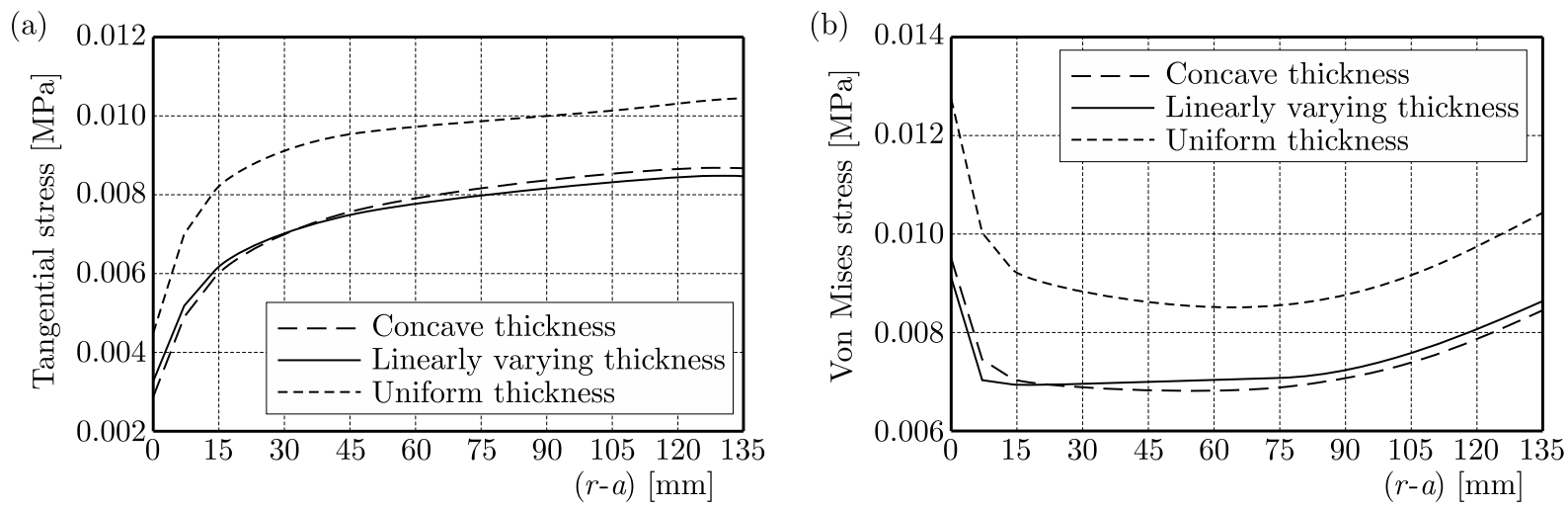

Fig. 5. Distribution of: (a) tangential stress, (b) von Mises stress

\section{Conclusion}

The present work proposes a study using the element based gradation of a varying material property of rotating disks and reports the stress and deformation behavior of uniform and variable thickness clamped rotating disks of exponentially graded FGMs. The element based grading of the material property yields an appropriate approach of functional grading as the shape functions in elemental formulations being co-ordinate functions make it easier to implement the same. The layered functional grading over a discrete area instead of elements, offers singularities in the field variables at adjoining lines or surfaces. The results obtained are found to be in good agreement with the established reports. Further, it is observed that varying geometry of FGM disks results into lower stress states in the disks and, hence, it can be concluded that variable thickness disks possess better strength than uniform disks of the same mass.

\section{References}

1. Afsar A.M., Go J., 2010, Finite element analysis of thermoelastic field in a rotating FGM circular disk, Applied Mathematical Modelling, 34, 3309-3320

2. Ali A., Bayat M., Sahari B.B., Saleem M., Zaroog O.S., 2012, The effect of ceramic in combinations of two sigmoid functionally graded rotating disks with variable thickness, Scientific Research and Essays, 7, 25, 2174-2188.

3. Bayat M., Sahari B.B., Saleem M., Dezvareh E., Mohazzab A.H., 2011, Analysis of functionally graded rotating disks with parabolic concave thickness applying an exponential function and the Mori-Tanaka scheme, IOP Conference Series: Materials Science and Engineering, 17, 1-11

4. Bayat M., Saleem M., Sahari B.B., Hamouda A.M.S., Mahdi E., 2009, Mechanical and thermal stresses in a functionally graded rotating disk with variable thickness due to radially symmetry loads, International Journal of Pressure Vessels and Piping, 86, 357-372

5. Callioglu H., 2011, Stress analysis in a functionally graded disc under mechanical loads and a steady state temperature distribution, Sadhana, 36, 53-64

6. Callioglu H., Bektas N.B., Sayer M., 2011a, Stress analysis of functionally graded rotating discs: analytical and numerical solutions, Acta Mechanica Sinica, 27, 950-955

7. Callioglu H., Sayer M., Demir E., 2011b, Stress analysis of functionally graded discs under mechanical and thermal loads, Indian Journal of Engineering and Material Sciences, 18, 111-118

8. Eraslan A.N., 2003, Elastic-plastic deformations of rotating variable thickness annular disks with free, pressurized and radially constrained boundary conditions, International Journal of Molecular Sciences, 45, 643-667 
9. Nejad A., Abedi M., Hassan M., Ghannad M., 2013, Elastic analysis of exponential FGM disks subjected to internal and external pressure, Central European Journal of Engineering, 3, 459-465

10. Rosyid A., SAheb M.E., Yahia F.B., 2014, Stress analysis of nonhomogeneous rotating disc with arbitrarily variable thickness using finite element method, Research Journal of Applied Sciences, Engineering and Technology, 7, 3114-3125

11. Seshu P., 2003, A Text Book of Finite Element Analysis, PHI Learning Pvt. Ltd.

12. Sharma J.N., Sharma D., Kumar S., 2012, Stress and strain analysis of rotating FGM Thermoelastic circular disk by using FEM, International Journal of Pure and Applied Mathematics, 74, $3,339-352$

13. Zafarmand H., Hassani B., 2014, Analysis of two-dimensional functionally graded rotating thick disks with variable thickness, Acta Mechanica, 225, 453-464

14. Zafarmand H., Kadkhodayan M., 2015, Nonlinear analysis of functionally graded nanocomposite rotating thick disks with variable thickness reinforced with carbon nanotubes, Aerospace Science and Technology, 41, 47-54

15. Kim J.H., Paulino G.H., 2002, Isoparametric graded finite elements for nonhomogeneous isotropic and orthotropic materials, ASME Journal of Applied Mechanics, 69, 502-514

Manuscript received June 1, 2016; accepted for print July 5, 2016 